\title{
Sensibilidad in vitro de cepas chilenas de Cryptococcus neoformans de origen clínico
}

\section{In vitro susceptibility of Chilean Cryptococcus neoformans strains of clinical source}

\author{
Rodrigo Cruz Ch. ${ }^{1}$, Peggy Vieille O. ${ }^{1}$ y Jana Stojanova ${ }^{2}$
}

'Laboratorio de Micología, Universidad de Valparaíso, Chile.

${ }^{2}$ Departamento de preclínicas, Escuela de Medicina, Universidad de Valparaíso, Chile.

Conflictos de Interés: Ninguno.

Financiamiento: Laboratorio de Micología Universidad de Valparaíso.

Recibido: 1 de septiembre de 2019 / Aceptado: 3 de marzo de 2020

\section{Resumen}

Introducción: Las infecciones por levaduras del género Cryptococcus afectan principalmente a pacientes con déficit de la inmunidad mediada por células. Han sido escasos los estudios de sensibilidad realizados para este género en Chile. Objetivos: Determinar la sensibilidad in vitro de Cryptococcus sp a antifúngicos de uso habitual y evaluar la concordancia esencial entre sensibilidad determinada por microdilución en caldo y por difusión en agar con tiras comerciales. Materiales y Método: Estudio descriptivo de 21 cepas aisladas desde liquido céfalo-raquídeo y sangre. Las $\operatorname{CIM}_{50} \mathrm{y}$ $\mathrm{CIM}_{90}$ para fluconazol, voriconazol y anfotericina $\mathrm{B}$ se determinaron por microdilución en caldo (Sensititre Yeast One ${ }^{\circledR}$ ) y por difusión en agar con tiras comerciales (MIC Test Strips). Resultados: Todas las cepas correspondieron a $C$. neoformans. Los rangos de $\mathrm{CIM}_{50}$ y CIM $_{90}$ para cada antifúngico estudiado fueron amplios por ambos métodos. La concordancia esencial entre microdilución y difusión en agar con tiras comerciales fue de 24,62 y $29 \%$ para fluconazol, voriconazol y anfotericina B, respectivamente. Conclusiones: La prueba de Sensititre Yeast One ${ }^{\circledR}$ y la de difusión en agar con tiras comerciales, MIC Test Strips, tienen una pobre concordancia esencial para fluconazol $\mathrm{y}$ anfotericina $\mathrm{B}$.

Palabras clave: Prueba de sensibilidad microbiana; antimicóticos; Cryptococcus neoformans; concentraciones inhibitorias mínimas.

\begin{abstract}
Background: Cryptococcus yeast infections primarily affect immunocompromised patients. There have been few susceptibility studies conducted for this genus in Chile. Aims: To determine the in vitro susceptibility to commonly used antifungals and evaluate the concordance between susceptibility determined by microdilution in broth and commercially available strips. Methods: Descriptive study of 21 Cryptococcus strains, isolated from cerebrospinal fluid and blood. The $\mathrm{MIC}_{50}$ and $\mathrm{MIC}_{90}$ for fluconazole, voriconazole and amphotericin B was determined by broth microdilution (Sensititre Yeast One ${ }^{\circledR}$ ) and by commercial drug sensitivity strips (MIC Test Strips). Results: All strains corresponded to C. neoformans. The ranges of $\mathrm{MIC}_{50}$ and $\mathrm{MIC}_{90}$ for each antifungal studied were wide by both methods. The essential agreement between Sensititre Yeast One test and strips was 24, 62 and $29 \%$ for fluconazole, voriconazole and amphotericin B, respectively. Conclusions: The Sensititre Yeast One test and MIC Test Strips exhibited poor essential concordance, especially for fluconazole and amphotericin B.

Keywords: Microbial sensitivity test; antifungal antibiotics; Cryptococcus neoformans; minimum inhibitory concentrations.
\end{abstract}


Cryptococcus aisladas de pacientes de la Región de Valparaíso, Chile.

a criptococosis es una infección fúngica sistémica provocada por levaduras del género Cryptococcus, las que son capsuladas y de amplia distribución mundial en distintos sustratos. Pueden causar enfermedades en paciente con inmunocompromiso, especialmente en aquellos con infección por VIH-SIDA ${ }^{1,2}$. Los humanos pueden adquirir estas levaduras principalmente por inhalación desde el medioambiente ${ }^{2}$.

Cryptococcus neoformans y C. gattii son los principales agentes etiológicos; sin embargo, otras especies como C. albidus, C. laurentii, C. adeliensis, C. curvatus y $C$. uniguttulatus también pueden provocar infecciones ocasionalmente ${ }^{3,4}$. Cryptococcus neoformans está constituido por los serotipos A (grubii) y D (neoformans), además de existir un hibrido AD. Cryptococcus gatti posee los serotipos $\mathrm{B}$ y $\mathrm{C}^{5}$. Estas especies tienen similares características morfológicas en medios no selectivos. La identificación molecular ha permitido clasificar a $C$. neoformans y $C$. gattii en ocho tipos, siendo $C$. neoformans var. grubii clasificado en los tipos moleculares VNI y VNII, la variedad neoformans en VNIII y VNIV y $C$. gattii como VGI, VGII, VGIII y VGIV ${ }^{4,5}$.

Crypyococcus neoformans se aísla, con frecuencia, desde suelos contaminados con deposiciones de palomas y de otras aves en las distintas regiones del mundo ${ }^{5,6}$, mientras que $C$. gattii se le ha asociado al árbol eucalipto en regiones tropicales y subtropicales del mundo; sin embargo, el brote en la isla de Vancouver demostró una asociación con otros árboles, incluyendo abetos y robles, además de un rango geográfico expandido de este agente ${ }^{5,6}$.

El tratamiento de estas infecciones incluye anfotericina B, fluconazol y 5-fluorocitocina; no obstante, han sido escasos los estudios de sensibilidad realizados para este género, especialmente en países como el nuestro. Esto se ha debido probablemente por la dificultad de la metodología de referencia y a la ausencia de puntos de corte clínicos bien establecidos ${ }^{7,8}$.

El Clinical Laboratory Standards Institute (CLSI) de E.U.A, en su documento M27-A permite medir las CIMs de las principales levaduras oportunistas (Candida spp, C. neoformans) demostrando una adecuada reproducibilidad inter-laboratorios ${ }^{8}$. También se ha desarrollado un estándar europeo, European Committee on Antimicrobial Susceptibility Testing (EUCAST), que en su documento EDef 7.1, ha demostrado ser equivalente al estándar CLSI en levaduras ${ }^{9}$.

El objetivo de este trabajo fue conocer la sensibilidad in vitro a los antifúngicos de uso habitual y la concordancia esencial entre los valores de CIM determinadas por microdilución en caldo y por difusión en agar con tiras comerciales de susceptibilidad, de cepas de

\section{Materiales y Método}

Estudio descriptivo de 21 cepas de Cryptococcus aisladas de líquido céfalo-raquídeo $(\mathrm{n}=17)$ y sangre $(n=4)$, de pacientes atendidos en los hospitales Carlos van Buren, Valparaíso y Gustavo Fricke, Viña del Mar.

\section{Identificación de especie}

La identificación se realizó por morfología de las colonias, microscopia, pruebas bioquímicas para la producción de melanina en agar Staib (alpiste negro 5\%, glucosa $0,1 \%$, creatinina $0,1 \%$, fosfato monopotásico $0,1 \%$, agar $2 \%$ ) y ureasa en agar urea de Christensen (Merck) a una temperatura de $35^{\circ} \mathrm{C}$, además de la prueba comercial de asimilación ID $32 \mathrm{C}$ (BioMérieux). También se sembraron en medio agar canavanina-glicina-bromotimol (CGB) para la búsqueda de $C$. gatti. Posteriormente, cada cepa fue sub-cultivada en agar Sabouraud para las correspondientes pruebas de sensibilidad antimicrobiana ${ }^{10,11}$.

\section{Estudio de sensibilidad}

Para microdilución en caldo se empleó el sistema comercial Sensititre Yeast One ${ }^{\circledR}$ (Trek Diagnostic Systems) para evaluar la CIM de fluconazol, voriconazol y anfotericina B, siguiendo las indicaciones del fabricante. El inóculo se preparó tomando dos a tres colonias jóvenes de 24 horas de incubación en agar Sabouraud, homogeneizando en $1 \mathrm{ml}$ de solución salina al 0,9\% hasta lograr una concentración Mc Farland de 1. De esta suspensión se transfirieron $20 \mu \mathrm{L}$ a un tubo que contenía $11 \mathrm{ml}$ de caldo Yeast One ${ }^{\circledR}$ obteniendo un inóculo de 1,5-8 x 10 $\mathrm{ufc} / \mathrm{ml}$. Las microplacas fueron inoculadas con $100 \mu \mathrm{L}$ por pocillo e incubadas a $35^{\circ} \mathrm{C}$ por $72 \mathrm{~h}$. Las CIM para azoles se leyeron visualmente (método colorimétrico) como la concentración más baja del antifúngico que inhibió el del pocillo control. Para anfotericina B, la CIM fue la concentración más baja que inhibió completamente el crecimiento en comparación con el crecimiento del control $^{12,13}$

Para la prueba de difusión en agar con tiras comerciales de sensibilidad se emplearon placas Petri de $90 \mathrm{~mm}$ de diámetro con $4 \mathrm{~mm}$ en altura con medio RPMI 1640 con L-glutamina, sin bicarbonato de sodio, con rojo fenol (Sigma Aldrich, Co.) suplementado con glucosa al 2\%, agar 1,5\% y tamponado con $165 \mathrm{mM}$ MOPS pH 7,0 (Sigma Co.). Se emplearon tiras comerciales MIC Test Strips (Liofielchem $^{\circledR}$ ) de fluconazol, voriconazol y anfotericina $\mathrm{B}$, siguiendo las recomendaciones del fabricante. Las placas fueron inoculadas e incubadas a $35^{\circ} \mathrm{C}$ por $48 \mathrm{~h}$. Las CIM fueron determinadas visualmente por halo de crecimiento en $50 \%$, en comparación con el crecimiento 


\begin{tabular}{|c|c|c|c|}
\hline & & Sensititre Yest One & MIC Test Strips \\
\hline \multirow[t]{4}{*}{ Fluconazol } & Concordancia esencial & \multicolumn{2}{|c|}{$6 / 21(29 \%)$} \\
\hline & Rango & $1-64$ & $0,075-256$ \\
\hline & $\mathrm{CIM}_{50}$ & 8 & 2 \\
\hline & $\mathrm{CIM}_{90}$ & 16 & 24 \\
\hline \multirow[t]{4}{*}{ Voriconazol } & Concordancia esencial & \multicolumn{2}{|c|}{$13 / 21(62 \%)$} \\
\hline & Rango & $0,015-1$ & $0,12-2$ \\
\hline & $\mathrm{CIM}_{50}$ & 0,06 & 0,094 \\
\hline & $\mathrm{CIM}_{90}$ & 0,25 & 0,852 \\
\hline \multirow[t]{4}{*}{ Anfootericina B } & Concordancia esencial & \multicolumn{2}{|c|}{$5 / 21(24 \%)$} \\
\hline & Rango & $0,12-1$ & $0,008-0,38$ \\
\hline & $\mathrm{CIM}_{50}$ & 0,25 & 0,047 \\
\hline & $\mathrm{CIM}_{90}$ & 0,5 & 0,225 \\
\hline
\end{tabular}

inhibición. Como cepa de control se utilizó Candida albicans ATCC $90028^{14,15}$.

\section{Análisis estadístico}

La concordancia esencial entre los dos métodos se consideró cuando la discrepancia en las CIM no superó dos $\log 2$ de diluciones de diferencia (es decir, no debió sobrepasar dos diluciones). Los valores de CIM para cada antifúngico y metodología fueron evaluadas usando STATA 15.0 (StataCorp) ${ }^{16}$. No evaluamos concordancia categórica debido a la falta de consenso en punto de ruptura para los antifungicos evaluados.

\section{Resultados}

De las 21 cepas estudiadas, todas correspondieron a C. neoformans. En la Tabla 1 se muestran los datos de

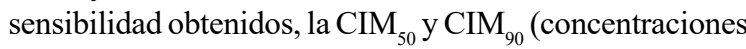
inhibitorias mínimas a la cual el 50 y $90 \%$ de las cepas fúngicas estudiadas detienen su crecimiento) para fluconazol, voriconazol y anfotericina $\mathrm{B}$, tanto por microdilución como por tiras comerciales. La concordancia esencial entre las dos metodologías fue baja para fluconazol y anfotericina B.

\section{Discusión}

Las infecciones por las distintas especies de Cryptococcus afectan principalmente a pacientes con algún grado de inmunocompromiso celular en las distintas regiones y países del mundo ${ }^{1,2,16}$. Cryptococcus neoformans es la especie más frecuentemente aislada en la mayoría de los países de América Latina, situación que coincide con nuestro trabajo ${ }^{1}$. La mayoría de los aislados se obtienen de líquido céfalo-raquídeo debido al importante tropismo de estas especies por el sistema nervioso central, lo que también coincidió con nuestros resultados, donde sólo cuatro cepas se obtuvieron de hemocultivos ${ }^{17-19}$.

En Chile han sido escasos los trabajos sobre sensibilidad a los antifúngicos que incluyan levaduras del género Cryptococcus $^{20}$. Este es el primer trabajo realizado sólo con cepas de $C$. neoformans aisladas de muestras médicas.

Debido a la complejidad en la preparación de las pruebas de microdilución en caldo (guía CLSI M-27), se han buscado métodos alternativos para implementar pruebas de sensibilidad de fácil preparación dentro de la rutina de los centros de diagnóstico microbiológico. El método comercial cuyo formato se asemeja más a la metodología del CLSI es Sensitre ${ }^{\circledR}$ Yeast One (TREK Diagnostic Systems), aprobado por la FDA, el que se basa en la microdilución en caldo, en presencia de un sustrato cromogénico para facilitar la interpretación de la $\mathrm{CIM}^{21,22}$. Varios trabajos han evaluado la equivalencia con el método estándar, demostrando que es una alternativa válida para determinar la CIM, tanto para levaduras del género Candida, como para Cryptococcus $\operatorname{spp}^{7,22,23,24}$.

Si bien, el CLSI ha estandarizado las pruebas de sensibilidad antifúngica empleando métodos de difusión en agar, únicamente para el género Candida ${ }^{25}$, Aller y cols., encontraron una concordancia de 81,1 y $89,2 \%$ para la sensibilidad de Cryptococcus a fluconazol y fluorocitocina entre Etest $^{\circledR}$, la prueba original por tiras comerciales, actualmente producidas por BioMérieux, y microdilución; sin embargo, fue de 8,1 y $13,5 \%$ para itraconazol y anfotericina $\mathrm{B}$, respectivamente ${ }^{26}$. Thomson y cols., encontraron una concordancia de $97 \%$ para la sensibilidad a isavuconazol entre Etest ${ }^{\circledR}$ y el método de referencia ${ }^{27}$. En nuestro estudio, se muestra que la concordancia entre los dos métodos fue solo de 24 y $29 \%$ para anfotericina B y fluconazol, respectivamente, antifúngicos que son los pilares en el tratamiento de estas infecciones ${ }^{28}$. Voriconazol ha demostrado tener buena actividad contra $C$. neoformans, por lo que puede ser una alternativa al tratamiento de elección; sin embargo, su mayor costo económico y un menor número de estudios clínicos han limitado su uso ${ }^{29}$. En nuestro estudio, sólo uno de los aislados con sensibilidad reducida a fluconazol $(\mathrm{CIM} \geq 16 \mu \mathrm{g} / \mathrm{ml})$ tuvo una CIM relativamente alta a voriconazol $(\mathrm{CIM}=1 \mu \mathrm{g} / \mathrm{ml})$.

Respecto a la correlación in vivo de los datos in vitro, hay pocas referencias en la literatura médica. En lo que se refiere a $C$. neoformans y fluconazol, Witt y cols., observaron que la probabilidad de un fallo terapéutico en casos de meningitis por Cryptococcus era mayor de $80 \%$ cuando la CIM era $\geq 16 \mu \mathrm{g} / \mathrm{ml}^{31}$. En nuestro trabajo, cinco cepas 
es necesario determinar CIM en caso de aislarse estas levaduras, ya que existen cepas con CIM elevada que pueden asociarse a fallo terapéutico en infecciones graves.

\section{Conclusiones}

Los rangos de $\mathrm{CIM}_{50}$ y $\mathrm{CIM}_{90}$ para fluconazol, voriconazol y anfotericina $\mathrm{B}$ fueron amplios por ambos métodos. La prueba de Sensititre Yeast One ${ }^{\circledR}$ y la de difusión en agar con tiras comerciales, MIC Test Strips, tienen una pobre concordancia esencial para fluconazol $\mathrm{y}$ anfotericina $\mathrm{B}$.

Agradecimientos: Gerardo Peralta del Hospital Carlos van Buren de Valparaíso y Alejandro Joyas del Hospital Gustavo Fricke de Viña del Mar por colaborar con cepas de sus centros.

\section{Referencias bibliográficas}

1.- Teodoro V L, Gullo F P, Sardi J de C, Torres E M, Fusco-Almeida A M, Mendes-Giannini MJ. Environmental isolation, biochemical identification, and antifungal drug susceptibility of Cryptococcus species. Rev Soc Bras Med Trop 2013; 46 (6): 759-64. doi: 10.1590/00378682-0025-2013.

2.- Chayakulkeeree M, Perfect J R. Cryptococcosis. Infect Dis Clin North Am 2006; 20: 507-44. doi:10.1016/j. idc.2006.07.001.

3.- Griffin A T, Hanson K E. Update on fungal diagnostics. Curr Infect Dis Rep 2014; 16: 415. doi: 10.1007/s11908-014-0415-z.

4.- Tapia C, Correa N. Género Cryptococcus. Rev Chil Infectol. 2014; 31 (6): 719-20. doi: 10182014000600012 .

5.- Vieille P, Cruz R, León P, Caceres N, Giusiano G. Isolation of Cryptococcus gattii VGIII from feline nasal injury. Med Mycol Case Rep. 2018; 22: 55-7. doi: 10.1016/jmmcr.2018.09.003.

6.- Kronstad J W, Attarian R, Cadieux B, Choi J, D'Souza C A, Griffiths E J, et al. Expanding fungal pathogenesis: Cryptococcus breaks out of the opportunistic box. Nat Rev Microbiol 2011; 9: 193-203. doi: 10.1038/nrmicro2522.

7.- García-Martos P, Noval J F, García-Tapia A, Marín P, Puerto J L, Sepúlveda A. Sensibilidad a antifúngicos de especies de Cryptococcus de interés clínico. Med Clin (Barc) 2002; 119 (6): 211-3. doi: 10.1016/S0025-7753(02)73366-6.

8. National Committee for Clinical Laboratory Standards. Reference method for broth dilution antifungal susceptibility testing of yeasts; approved standard NCCLS document M27-A2. Wayne, PA: National Committee for Clinical
Laboratory Standard; 2017. https://clsi.org/ standards/products/microbiology/documents/ $\mathrm{m} 27 /$.

9.- Subcommittee on Antifungal Susceptibility Testing (AFST) of the ESCMID European Committee for Antimicrobial Susceptibility Testing (EUCAST). EUCAST definitive document EDef 7.1: method for the determination of broth dilution MICs of antifungal agents for fermentative yeasts. Clin Microbiol Infect 2008; 14: 398-405. doi: 10.1111/j.1469-0691.2007.01935.x.

10.- Kwon-Chung K J, Polacheck I, Bennett J E. Improved diagnostic medium for separation of Cryptococcus neoformans var. neoformans (serotypes A and D) and Cryptococcus neoformans var. gattii (serotypes B and C) J. Clin. Microbiol 1982; 15 (3): 535-7. https:/www.ncbi.nlm.nih.gov/pmc/articles/ PMC272134/.

11.- Staib F, Seibold M, Antweiler E, Frohlich B, Weber S, Blisse A. The brown colour effect (BCE) of Cryptococcus neoformans in the diagnosis, control and epidemiology of C. neoformans infections in AIDS patients. Zent. Bakteriol. Mikrobiol. Hyg A 1987; 266 (1-2): 167-77. doi: 10.1016/s01766724(87)80030-5.

12.- Pfaller M A, Barry A L. Evaluation of a novel colorimetric broth microdilution method for antifungal susceptibility testing of yeast isolates. J Clin Microbiol 1994; 32: 1992-96. https://www.ncbi.nlm.nih.gov/ pubmed/7989555.

13.- Pfaller M A, Vu Q, Lancaster M, EspinelIngroff A, Fothergill A, Grant C, et al. Multisite reproducibility of colorimetric broth microdilution method for antifungal susceptibility testing of yeast isolates. J Clin Microbiol 1994; 32: 1625-8. PMC263742.

14.- MIC Test Strip. Paper strips for determining the Minimum Inhibitory Concentration (M.I.C.) https://www.liofilchem.com/en-us/products/ featured-products/mic-test-strip-liofilchem. html. Consultado 19/02/2020.

15.- https://www.stata.com/stata15/. Consultado $19 / 02 / 2020$

16.- Firacative C, Lizarazo J, Illnait-Zaragozí M T, Castañeda E, Latin American Cryptococcal Study Group. The status of cryptococcosis in Latin America. Mem Inst Oswaldo Cruz 2018; 113 (7): e170554. doi: 10.1590/007402760170554 .

17.- Cortés J, Kral A, Wilson G. Criptococcosis en el Hospital Carlos Van Buren de Valparaíso: una serie de casos. Rev Chilena Infectol. 2018; 35 (4): 420-23. doi: 10.4067/s071610182018000400420 .

18.- Andrade-Silva L, Ferreira-Paim K, Mora D J, da Silva P R, Andrade A A, Araujo N E, et al. Susceptibility profile of clinical and environmental isolates of Cryptococcus neoformans and Cryptococcus gattii in Uberaba, Minas Gerais, Brazil. Med Mycol. 2013; 51(6): 635-40. doi: 10.3109/13693786.2012.761737.

19.- Canessa J C, Cabrera D, Eskenazi J, Samalvides F. Associated factors for inhospital mortality in patients with meningeal cryptocococosis and HIV infection at a local hospital in Lima, Peru. World J AIDS 2011; 1 (1): 8-14. doi: 10.4236/wja.2011.11002.

20.- Silva V, Díaz C, Febré N. Vigilancia de la resistencia de levaduras a antifúngicos. Rev Chil Infect 2002; 19 (2): 149-56. doi: $10.4067 /$ S0716-10182002019200016. 
21.- FDA. 510(k) Premarket Notification. Susceptibility Test Plate. Antifungal. https:// www.accessdata.fda.gov/scripts/cdrh/cfdocs/ cfpmn/pmn.cfm?ID=K133038. Acceso 15 Junio 2019.

22.- Archibald L K, Tuohy M J, Wilson D A, Nwanyanwu O, Kazembe P N, Tansuphasawadikul S, et al. Antifungal susceptibilities of Cryptococcus neoformans. Emerg Infect Dis 2004; 10 (1): 143-5. doi: 10.3201/eid1001.020779.

23.- Pfaller M A, Chaturvedi V, Diekema D J , Ghannoum M A, Holliday N M, Killian S $\mathrm{B}$, et al. Clinical evaluation of the Sensititre YeastOne colorimetric antifungal panel for antifungal susceptibility testing of the echinocandins anidulafungin, caspofungin, and micafungin. J Clin Microbiol 2008; 46 (7): 2155-9. doi: 10.1128/JCM.00493-08.

24.- Espinel-Ingroff A, Pfaller M, Messer S A, Knapp C C, Killian S, Norris H A, et al. Multicenter comparison of the Sensititre YeastOne colorimetric antifungal panel with the National Committee for Clinical Laboratory Standards M27-A reference method for testing clinical isolates of common and emerging Candida spp., Cryptococcus spp., and other yeasts and yeast-like organisms. J Clin Microbiol 1999; 37( 3): 591-5. https://www. ncbi.nlm.nih.gov/pmc/articles/PMC84481/pdf/ jm000591.pdf.

25.- Alexander B D, Byrne T C, Smith K L, Hanson K E, Anstrom K J, Perfect J R, et al. Evaluación comparativa de los paneles Etest y Sensititre YeastOne frente al método de microdilución del caldo de referencia del Instituto de Estándares Clínicos y de Laboratorio M27-A2 para evaluar la susceptibilidad de Candida a siete agentes antimicóticos. J Clin Microbiol 2007; 45 (3): 698-706. doi: 10.1128/JCM.01840-06.

26.- Aller A I, Martín-Mazuelos E, Gutiérrez M J, Bernal S, Chávez M, Recio F J. Comparison of the Etest and microdilution method for antifungal susceptibility testing of Cryptococcus neoformans to four antifungal agents. J Antimicrob Chemother 2000; 46 (6): 997-1000. doi: 10.1093/jac/46.6.997.

27.- Thompson G R, Fothergill A W, Wiederhold N P, Vallor A C, Wickes B L, Patterson T F. Evaluation of Etest method for determining isavuconazole MICs against Cryptococcus gattii and Cryptococcus neoformans. Antimicrob Agents Chemother 2008; 52 (8): 2959-61. doi: 10.1128/AAC.00646-08.
28.- Abassi M, Boulware D, Rhein J. Cryptococcal meningitis: diagnosis and management update. Curr Trop Med Rep 2015; 2(2): 90-9. doi: 10.1007/s40475-015-0046-y.

29.- Govender N, Patel J, van Wyk M, Chiller T, Lockhart S, for the Group for Enteric, Respiratory and Meningeal Disease Surveillance in South Africa (GERMS-SA). Trends in antifungal drug susceptibility of Cryptococcus neoformans isolates obtained through population-based surveillance in South Africa in 2002-2003 and 2007-2008. Antimicrob Agents Chemother 2011; 55 (6): 2606-26. doi: 10.1128/AAC.00048-11.

30.- Witt M D, Lewis R J, Larsen R A, Milefchik E $\mathrm{N}$, Leal M A, Haubrich R H, et al. Identification of patients with acute AIDS-associated cryptococcal meningitis who can be effectively treated with fluconazole: the role of antifungal susceptibility testing. Clin Infec Dis 1996; 22 (2): 322-8. doi: 10.1093/clinids/22.2.322.

31.- Meng L, Yong L, Min C, Weihua P, Lixing W. Antifungal susceptibilities of Cryptococcus species complex isolates from AIDS and nonAIDS patients in Southeast China. Braz J Infect Dis 2012 ; 16 (2): 175-9. doi: 10.1016/S14138670(12)70301-X. 